\section{A missão da RBP na Educação Médica Continuada}

\author{
The RBP's mission in \\ the Continuing Medical \\ Education
}

A Revista Brasileira de Psiquiatria (RBP) tem a dupla missão de incentivar a Educação Médica Continuada dos seus leitores e de divulgar achados de pesquisa. Para tanto, publicamos, em português, artigos de revisão e de atualização (inclusive em suplementos temáticos) e, em inglês, artigos originais e comunicações breves. Dessa forma, acreditamos levar ao associado da Associação Brasileira de Psiquiatria (ABP) o que existe de mais atual no diagnóstico, fisiopatologia e tratamento dos principais transtornos psiquiátricos.

Nos últimos cinco anos, a RBP publicou 24 artigos de atualização clínica e 12 de revisão, além de 11 suplementos contendo 94 artigos, o que totaliza 130 artigos. Os temas dessas publicações abrangeram os transtornos psiquiátricos mais prevalentes (transtornos do humor, transtornos ansiosos e dependência de substâncias) e subespecialidades (psiquiatria infantil e geriátrica). Entre as diretrizes de tratamento - publicadas como artigos de revisão destacam-se a "Diretriz da Associação Brasileira de Estudos Sobre Álcool e Outras Drogas (ABEAD) para o Diagnóstico e Tratamento das Comorbidades Psiquiátricas Associadas Dependência de Álcool e Outras Drogas" e "Consenso brasileiro sobre transtornos metabólicos e antipsicóticos de segunda geração".

Fizemos um levantamento sobre a abrangência das áreas abordadas nos artigos relevantes para a prática clínica, assim como os artigos mais acessados no site da RBP e pela SciELO. Entre os artigos de revisão e atualização publicados nos últimos cinco anos, percebemos um destaque para as seguintes áreas: transtornos do humor, transtornos da ansiedade, psiquiatria infantil, abuso e dependência de álcool e outras substâncias, neuropsiquiatria e psiquiatria geriátrica. Os suplementos sobre abuso e dependências de substâncias e transtorno afetivo bipolar foram as publicações mais acessadas pela Internet, recebendo 151.792 e 131.128 requisições de acesso, respectivamente. Entre os 25 artigos com maior número de requisições de download entre 2003 e 2008, 15 são artigos de suplementos, três são artigos de revisão e dois são atualizações, somando 306.375 downloads. Esses dados mostram o grande interesse dos leitores da revista em receber atualizações sobre os transtornos de maior prevalência na população.

O corpo editorial da RBP julga estar cumprindo a sua tarefa de oferecer artigos de interesse para clínicos com temas atuais e relevantes para a prática. A melhora da qualidade da revista (inclusive do Fator de Impacto) tem possibilitado que autores internacionais de altíssimo prestígio escrevam revisões e atualizações para a RBP, colocando à disposição dos leitores o melhor da literatura internacional. Um exemplo disto é a presença de Aaron Beck no suplemento sobre terapia cognitivo-comportamental, publicado em 2008.

No entanto, chama a atenção a carência em certas áreas como a de interconsultas (tema de apenas um artigo de revisão nos últimos cinco anos), suicídio (ausência de artigos de revisão) e psiquiatria forense (tema de apenas um suplemento). As atualizações sobre esses assuntos são de interesse dos leitores da revista, mas as submissões de revisões ainda são pouco freqüentes. Além disso, os editores consideram que suplementos sobre temas muito im- 
portantes, como depressão e esquizofrenia, publicados há mais de cinco anos, merecem uma versão atualizada e já estão empenhados nesta missão. A publicação do próximo suplemento de atualização sobre depressão está prevista para 2009.

Sendo assim, os editores da RBP convidam os autores a submeterem atualizações clínicas, e seguirão trabalhando para que os associados da ABP recebam sempre informações atualizadas, escritas por especialistas, sobre diagnóstico e tratamento das condições psiquiátricas mais freqüentes nas suas práticas clínicas.

Beny Lafer, Juliana Belo Diniz Departamento de Psiquiatria, Universidade de São Paulo (USP), São Paulo (SP), Brasil

Guilherme V. Polanczyk

Departamento de Psicologia e Neurociência, Duke University, EUA
Leonardo F. Fontenelle

Departamento de Psiquiatria e Medicina Legal, Instituto de Psiquiatria (IPUB), Universidade Federal do Rio de Janeiro (UFRJ), Rio de Janeiro (RJ), Brasi

Departamento de Psiquiatria e Saúde Mental, Instituto de Saúde da Comunidade (ISC), Universidade Federal Fluminense

(UFF), Niterói (RJ), Brasil

Hermano Tavares

Instituto de Psiquiatria, Hospital das Clínicas, Faculdade de Medicina, Universidade de São Paulo (USP), São Paulo (SP), Brasil

Marcelo Pio de Almeida Fleck Departamento de Psiquiatria, Universidade Federal do Rio Grande do Sul (UFRGS), Porto Alegre (RS), Brasil

Rodrigo Affonseca Bressan Departamento de Psiquiatria, Universidade Federal de São Paulo (Unifesp), São Paulo (SP), Brasil

Financiamento e conflito de interesses

\begin{tabular}{|c|c|c|c|c|c|c|c|}
\hline $\begin{array}{l}\text { Membro do } \\
\text { grupo de } \\
\text { autores }\end{array}$ & $\begin{array}{l}\text { Local de } \\
\text { trabalho }\end{array}$ & $\begin{array}{l}\text { Verba de } \\
\text { pesquisa }\end{array}$ & $\begin{array}{l}\text { Outro apoio à pesquisa } \\
\text { ou educação médica } \\
\text { continuada }\end{array}$ & $\begin{array}{l}\text { Honorários de } \\
\text { palestrante }\end{array}$ & $\begin{array}{l}\text { Participação } \\
\text { acionária }\end{array}$ & $\begin{array}{l}\text { Consultorl } \\
\text { Conselho } \\
\text { consultivo }\end{array}$ & Outro $^{3}$ \\
\hline Beny Lafer & USP** & $\begin{array}{l}\text { FAPESP***} \\
\text { CNPq }^{* * *}\end{array}$ & $\begin{array}{c}\text { APA/AstraZeneca Young } \\
\text { Minds in Psychiatry } \\
\text { International Awards }\end{array}$ & Astra-Zeneca* & $\cdots$ & $\cdots$ & $\cdots$ \\
\hline $\begin{array}{l}\text { Juliana Belo } \\
\text { Diniz }\end{array}$ & USP & $\begin{array}{l}\text { FAPESP }^{* * *} \\
\text { CNPq }\end{array}$ & Novartis & $\cdots$ & $\cdots$ & $\cdots$ & Jansen-Cliag \\
\hline $\begin{array}{l}\text { Leonardo F. } \\
\text { Fontenelle }\end{array}$ & $\begin{array}{l}\text { UFRJ } \\
\text { UFF }\end{array}$ & $\begin{array}{l}\text { CNPq }^{* * *} \\
\text { FAPERJ } * * *\end{array}$ & $\begin{array}{l}\text { Bolsa Produtividade } \\
\text { CNPq }^{*}\end{array}$ & Eli-Lilly* & $\cdots$ & $\cdots$ & $\begin{array}{l}\text { Lundbeck* } \\
\text { Servier* } \\
\text { Editora } \\
\text { Guanabara- } \\
\text { Koogan }\end{array}$ \\
\hline $\begin{array}{l}\text { Guilherme V. } \\
\text { Polanczyk }\end{array}$ & $\begin{array}{c}\text { Duke } \\
\text { University }\end{array}$ & $\begin{array}{l}\text { NIMH }^{* * *} \\
\text { MRC }^{* * *} \\
\text { HRC }^{* * *} \\
\text { CNPq }^{* * *} \\
\text { NARSAD }^{* *}\end{array}$ & $\begin{array}{l}\text { Novartis }{ }^{* * *} \\
\text { Eli-Lilly** } \\
\text { Janssen- } \\
\text { Cilag }{ }^{* * *} \\
\text { Abbott*** } \\
\text { Shire*** }\end{array}$ & Novartis $^{*}$ & $\cdots$ & $\cdots$ & $\cdots$ \\
\hline $\begin{array}{l}\text { Hermano } \\
\text { Tavares }\end{array}$ & $\begin{array}{l}\text { USP* } \\
\text { ANJOTI* }\end{array}$ & FAPESP** & $\cdots$ & $\begin{array}{l}\text { Janssen-Cilag* } \\
\text { Instituto Américo } \\
\text { Bairral }\end{array}$ & $\cdots$ & $\cdots$ & $\begin{array}{c}\text { Lundbeck* } \\
\text { Servier* } \\
\text { Roche* } \\
\text { Apsen* } \\
\text { Wyeth* } \\
\text { Sandoz* } \\
\text { Abbott* } \\
\text { Cristália } \\
\text { Editora Artes } \\
\text { Médicas } \\
\text { TAM Linhas } \\
\text { Aéreas }\end{array}$ \\
\hline $\begin{array}{l}\text { Marcelo Pio de } \\
\text { Almeida Fleck }\end{array}$ & $\begin{array}{l}\text { UFRGS } \\
\text { HCPA }\end{array}$ & $\begin{array}{c}\text { CNPq*** } \\
\text { FIPE/HCPA }{ }^{* * *} \\
\text { Sixth Framework } \\
\text { Programm: } \\
\text { Comunidade } \\
\text { Européia* }\end{array}$ & $\begin{array}{l}\text { Bolsa Produtividade } \\
\mathrm{CNPq}^{*}\end{array}$ & $\cdots$ & $\cdots$ & $\begin{array}{c}\text { Consultor } \\
\text { temporário } \\
\text { da } \\
\text { Organização } \\
\text { Mundial da } \\
\text { Saúde }\end{array}$ & $\cdots$ \\
\hline $\begin{array}{l}\text { Rodrigo } \\
\text { Affonseca } \\
\text { Bressan }\end{array}$ & $\begin{array}{l}\text { UNIFESP** } \\
\text { Instituto } \\
\text { Albert } \\
\text { Einstein de } \\
\text { Ensino e } \\
\text { Pesquisa*** }\end{array}$ & $\begin{array}{c}\text { CNPq }^{* * *} \\
\text { FAPESP*** } \\
\text { Instituto Albert } \\
\text { Einstein de Ensino } \\
\text { e Pesquisa*** }\end{array}$ & $\begin{array}{c}\text { Novartis }{ }^{* * *} \\
\text { Eli-Lilly }{ }^{* * *} \\
\text { Janssen-Cilag***} \\
\text { Astra-Zeneca*** }\end{array}$ & $\begin{array}{c}\text { Novartis* } \\
\text { Eli-Lilly* } \\
\text { Janssen-Cilag* } \\
\text { Astra-Zeneca* }\end{array}$ & $\cdots$ & $\begin{array}{l}\text { Astra- } \\
\text { Zeneca* } \\
\text { Janssen- } \\
\text { Cilag* }^{*}\end{array}$ & $\begin{array}{l}\text { Astra-Zeneca* } \\
\text { Eli-Lilly* } \\
\text { Janssen-Cilag* }\end{array}$ \\
\hline
\end{tabular}

- Modesto

- Significativa

-.. Significativa. Montantes fornecidos à instituição do autor ou a colega para pesquisa onde o autor tem participação, não diretamente ao autor.

Nota: USP = Universidade de São Paulo; UFRJ = Universidade Federal do Rio de Janeiro; UFF = Universidade Federal Fluminense; ANJOTI = Associação Nacional

do Jogo Patológico e Outros Transtornos do Impulso; UFRGS = Universidade Federal do Rio Grande do Sul; $\mathrm{HCPA}=\mathrm{Hospital}$ de Clínicas de Porto Alegre;

UNIFESP = Universidade Federal de São Paulo; FAPESP = Fundação de Amparo à Pesquisa de São Paulo; CNPq = Conselho Nacional de Desenvolvimento

Cientifico e Tecnológico; FAPERJ = Fundação de Amparo à Pesquisa do Estado do Rio de Janeiro; NIMH = National Institute of Mental Health; MRC = Medical

Research Council; HRC = Humane Research Council; NASARD = National Alliance for Research on Schizophrenia and Depression; FIPE/HCPA = Fundo de

Incentivo à Pesquisa do Hospital de Clínicas de Porto Alegre.

Para mais informaçōes, consultar Instruções aos autores. 